OPEN ACCESS

Edited by:

Johanna Palmio,

University of Tampere, Finland

Reviewed by:

Fiore Manganelli,

University of Naples Federico II, Italy

Miriam Laura Fichtner,

Yale Medicine, United States

*Correspondence:

Weixi Zhang

zhangwxi@mail.sysu.edu.cn

Specialty section:

This article was submitted to Neuromuscular Disorders and

Peripheral Neuropathies,

a section of the journal

Frontiers in Neurology

Received: 15 June 2021

Accepted: 25 October 2021

Published: 01 December 2021

Citation:

Feng $X$, Song Z, WU M, LiU Y, Luo S,

Zhao $C$ and Zhang W (2021) Efficacy

and Safety of Immunotherapies in

Refractory Myasthenia Gravis: A

Systematic Review and Meta-Analysis.

Front. Neurol. 12:725700.

doi: 10.3389/fneur.2021.725700

\section{Efficacy and Safety of Immunotherapies in Refractory Myasthenia Gravis: A Systematic Review and Meta-Analysis}

\author{
Xuelin Feng 1,2, Zubiao Song ${ }^{2}$, Mengli $\mathrm{Wu}^{2}$, Yanmei Liu ${ }^{2}$, Sushan $\mathrm{Luo}^{3}$, Chongbo Zhao ${ }^{3}$ \\ and Weixi Zhang ${ }^{2 *}$
}

${ }^{1}$ Department of Neurology, East Hospital, Tongji University School of Medicine, Shanghai, China, ${ }^{2}$ Department of Neurology, National Key Clinical Department and Key Discipline of Neurology, The First Affiliated Hospital, Sun Yat-sen University,

Guangzhou, China, ${ }^{3}$ Department of Neurology, Huashan Hospital, Fudan University, Shanghai, China

Introduction: Approximately 10-20\% of patients WITH myasthenia gravis (MG) are refractory to conventional immunotherapies. The purpose of this study was to conduct a systematic review and meta-analysis to explore the optimal therapies for refractory MG.

Method: Correlative studies were performed through a search in PubMed, Cochrane Library, and Embase databases. The primary outcome was defined by changes in the quantitative myasthenia gravis score (QMG). Secondary outcomes were defined by the Myasthenia Gravis Activities of Daily Living Scale (MG-ADL), Myasthenia Gravis Foundation of America (MGFA) post intervention status, adverse events, and disease exacerbation after treatment.

Result: A total of 16 studies were included with 403 patients with refractory MG on therapies with rituximab, eculizumab, tacrolimus, and cladribine. Therapeutic efficacy of rituximab and eculizumab was identified with an estimated reduction in QMG score (4.158 vs. 6.928) and MG-ADL (4.400 vs. 4.344), respectively. No significant changes were revealed in efficacy or exacerbation density between the two independent therapeutic cohorts. The estimated adverse event density of eculizumab was more significant than that in the rituximab group (1.195 vs. 0.134 per patient-year), while the estimated serious event density was similar.

Conclusion: The efficacy and safety of rituximab and eculizumab have been approved in patients with refractory MG. Rituximab had a superior safety profile than eculizumab with a lower incidence of adverse events.

\section{Systematic Review Registration: https://www.crd.york.ac.uk/prospero/ display_record.php?ID=CRD42021236818, identifier CRD42021236818.}

Keywords: refractory myasthenia gravis, immunotherapies, tacrolimus, eculizumab (monoclonal antibody to $\mathrm{C5}$ ), rituximab-ofatumumab-ibrutinib-idelalisib

\section{INTRODUCTION}

Myasthenia gravis (MG) is an autoimmune disorder due to a transmission defect in the neuromuscular junction. These patients clinically manifest with fluctuating muscle weakness in an ocular, limb, and axial muscles. The majority of patients with MG have excellent responses to acetylcholinesterase inhibitors, rescue therapies (such as intravenous immunoglobulin and 
plasma exchange), immunosuppressants including glucocorticoid, azathioprine, mycophenolate mofetil, and thymectomy (1). However, still, a substantial proportion of patients have poor responses to conventional immunotherapies, termed refractory MG. Patients with refractory MG are usually managed with long-term high-dose glucocorticoid or immunosuppressive agents, which are associated with severe adverse events (2). In the recent decades, emerging clinical trials have been conducted using cyclophosphamide, rituximab, and eculizumab (3-5) to treat patients with refractory MG. Currently, the guidelines for therapeutic options in refractory MG have not been adequately established in clinical practice. In this study, we performed a meta-analysis to evaluate and compare the efficacy and safety of immunotherapies for patients with refractory MG.

\section{METHODS}

\section{Protocol Registration}

The meta-analysis protocol has been registered in PROSPERO (International Prospective Register of Systematic Reviews) under ID: CRD42021236818.

\section{Search Strategy}

One author (FX) performed an article search in PubMed, Cochrane Library, and Embase. Medical subject headings $(\mathrm{MeSH})$ and free-text words were used for PubMed and Cochrane: Myasthenia Gravis/therapy (MeSH) and refractory. In Embase, Emtree, and free-text words were used: MG (Emtree), therapy (Emtree), and refractory. The primary search was completed on December 16, 2020 and the final search was completed on March 15, 2021. Only English language papers were included.

\section{Study Selection}

Randomized controlled trials and observational studies with at least 10 patients with MG with each intervention were included. Refractory MG was defined according to criteria of Mantegazza (6): 1. Patients have an insufficient response to maximal safe doses of steroids and at least one immunosuppressive drug at an adequate dose and duration, 2. Inability to reduce immunosuppressive therapy without clinical relapse or a need for ongoing rescue therapy such as intravenous immunoglobulin or plasma exchange, 3. Severe or intolerable adverse effects from immunosuppressive therapy, 4 . Comorbid conditions that restrict the use of conventional therapies, 5. Frequent myasthenic crises even while on therapy. Exclusion criteria included: 1. Studies that did not have a clear standard of refractory MG or failed to meet criteria of Mantegazza, 2. Studies that did not evaluate the efficacy of specific therapy with a quantitative outcome, 3. Studies that were restricted to ocular MG or juvenile MG. Included studies were first filtered based on the title and abstract. Further assessment for eligibility was based on the full text of the studies (PRISMA flow chart; Figure 1).

\section{Quality Evaluation}

Newcastle-Ottawa Scale (NOS) was applied for quality evaluation for observational studies (7). According to the Cochrane
Handbook, randomized clinical trials and non-randomized controlled studies were assessed (8). Two authors (FX and SZ) independently evaluated the quality of the included studies, and an open discussion would be held to resolve disagreements.

\section{Data Extraction}

Two authors (FX and WM) independently extracted data. The discussion aimed to resolve any disagreements until a consensus was reached, or by consulting a third author (ZW). The following data were extracted: author, year of publication, country, original inclusion criteria, the total number of patients included in the study, intervention, related quantitative outcomes, and adverse events. Serious adverse events include adverse events that are lifethreatening or result in death, hospitalization, or persistent or significant disability or incapacity, are congenital anomalies or birth defects, or are essential medical events (9). Worsening of MG and MG crisis were not considered as serious adverse events.

\section{Outcome Definition}

The primary outcome was the changes in the quantitative myasthenia gravis score (QMG) (10). Secondary outcomes include Myasthenia Gravis Composite (MGC) scale (11), manual muscle test (MMT) (12), Myasthenia Gravis Activities of Daily Living (MG-ADL) scale (13), Myasthenia Gravis Foundation of America (MGFA) post intervention status (PIS) (14), adverse events and exacerbation of MG (defined as MG symptoms with increased frequency and/or intensity), and myasthenic crisis (defined as exacerbated MG symptoms that required intubation or rescue therapy such as plasma exchange and intravenous immunoglobulin) during treatment.

\section{Statistical Analysis}

Statistical analysis was based on STATA (version 14 for Windows). If some of the included studies failed to provide the SD of the change of reported outcomes, we would calculate it based on the methods described by The Cochrane Collaboration in the Cochrane Handbook for Systematic Reviews of Interventions (section 6.5.2.8, the correlation coefficient was calculated from similar studies) (8). Q-test and $I^{2}$-statistics were used for heterogeneity analysis. Fixed effect model or random effect model will be used based on heterogeneity (random effect model will be used if $I^{2}>50 \%$ ). Publication bias was explored with Egger's test.

\section{RESULTS}

\section{Search and Selection Results}

Our search in Embase, PubMed, and Cochrane library provided 626 studies. After deduplication, 504 studies were screened for the title and abstract. Full-text assessments were then performed for 75 studies, and we subsequently included 23 studies. Among them, seven studies did not report main outcomes, additional outcomes, or other quantitative outcomes of treatment effect. Therefore, 16 studies finally entered the quantitative synthesis (5, 15-26) (Figure 1). Of these included studies, 13 were observational studies and two were randomized controlled trials. The remaining was an open-label extension of other included 


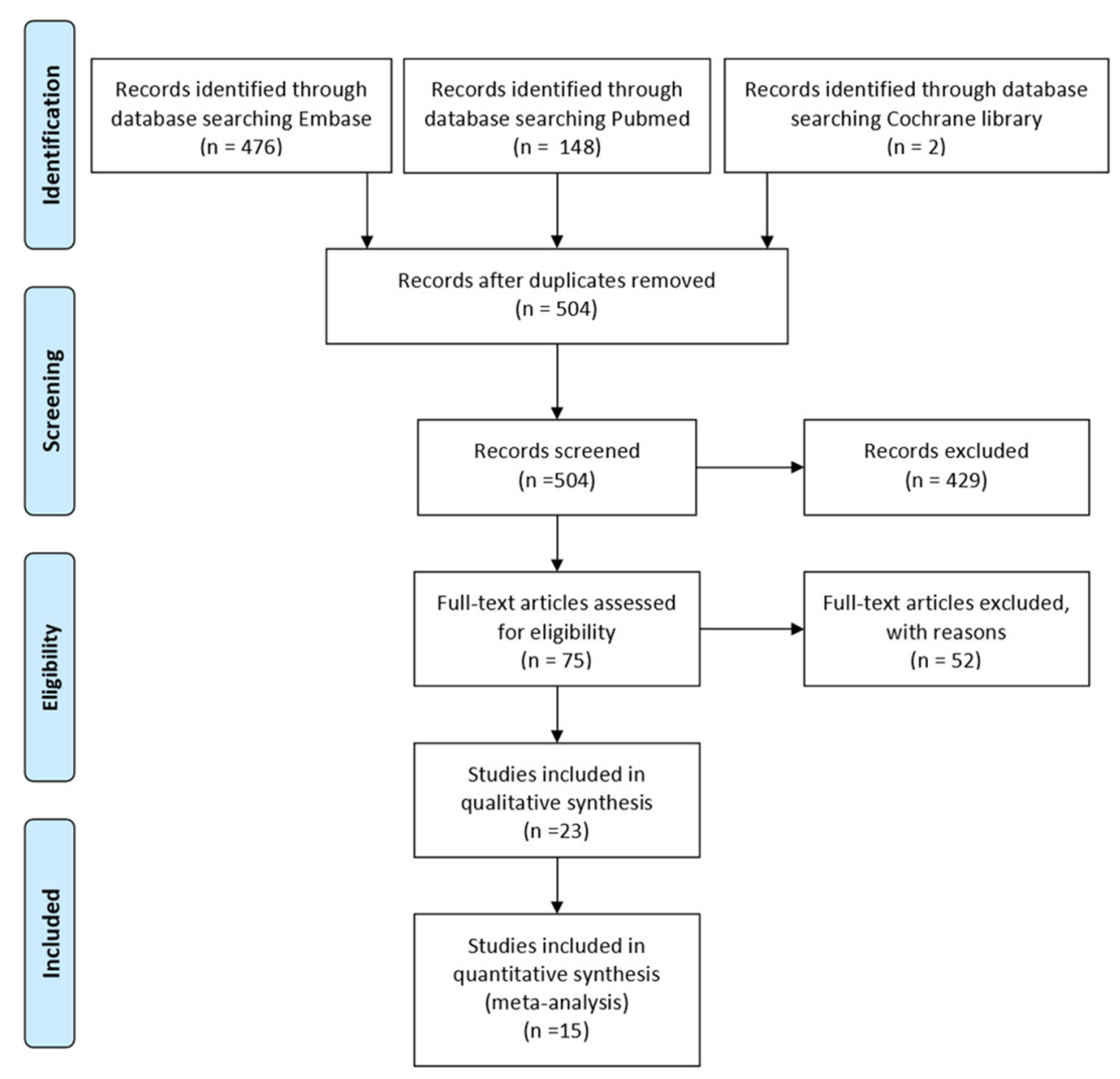

FIGURE 1 | The preferred reporting items for systematic reviews and meta-analyses (PRISMA) flow chart of studies selection.

studies (17). Quality evaluation of the included studies was in Supplementary Table 1. A summary of all the full-text reviewed studies was in Supplementary Table 2.

\section{Overall Features of Included Studies}

In the included studies, eight studies were on rituximab and five studies were on eculizumab, while two studies were about tacrolimus and cladribine, respectively (Table 1). In these studies, 403 patients (254 females and 149 males) were included (Table 2). The mean age of overall patients at recruitment was $48.4 \pm$ 19.06 years. The mean age of the patients in studies categorized by different therapies was $49.3 \pm 15.97$ (rituximab), $48.6 \pm$ 16.59 (eculizumab), $43.8 \pm 13.20$ (tacrolimus), and $60.8 \pm 18.59$ (cladribine), respectively. The mean age of the cladribine group was significantly higher than rituximab $(p=0.0069$, Bonferroni corrected) and tacrolimus group ( $p=0.0013$, Bonferroni corrected). There were 339 cases with the antiacetylcholine receptor (AChR) antibody positive and 43 cases with musclespecific tyrosine kinase (MuSK) antibody positive. All the cases with positive anti-MuSK antibodies were recruited in studies about rituximab. The remaining 21 patients were seronegative for MG-related antibodies.

\section{Efficacy in Ameliorating Disease Severity}

In the included studies, eight studies provided the primary outcome data in the QMG score (Figure 2A). The study by Jing et al. failed to provide the SD of the change of QMG score. It was imputed with the average correlation coefficients of LandonCardinal et al., and Brauner et al. Random effect model was used for quantitative synthesis. The pooled mean difference of rituximab was 4.158 (95\% CI: 2.994-5.323). The pooled mean difference of tacrolimus was 5.400 (95\% CI: 3.350-7.450). The pooled mean difference of eculizumab was 6.928 (95\% CI: 3.04210.813). No small-study effect was found by Egger's test ( $p$ $=0.281$ ).

Two of the included studies provided an MGC scale (20, 27), and one included a study that provided MMT other than QMG score (28). Combined with these studies, a standard mean difference in Glass's $\Delta$ was calculated in the random effect model (Figure 2B). The estimated standard mean difference of rituximab was 1.719 (95\% CI: 0.453-2.985). The estimated 
TABLE 1 | Summary of all the selected studies.

\begin{tabular}{|c|c|c|c|c|c|c|c|c|}
\hline No. & Author & Year & Therapy & $N$ & AChR-ab + & Musk-ab + & Seronegative & Outcomes \\
\hline 1 & H. Wu & 2020 & Tacrolimus & 24 & 18 & 0 & 6 & QMG, MG-ADL \\
\hline 2 & M. Oyama & 2020 & Eculizumab & 11 & 11 & 0 & 0 & QMG, MGFA-PIS, MG-ADL \\
\hline 4 & J. Howard* & 2017 & Eculizumab & 62 & 62 & 0 & 0 & QMG, MG-ADL \\
\hline 5 & R. Mantegazza* & 2021 & Eculizumab & 117 & 117 & 0 & 0 & MGFA-PIS \\
\hline 8 & D. Anderson & 2016 & Rituximab & 14 & 5 & 6 & 3 & MMT \\
\hline 9 & k. Choi & 2019 & Rituximab & 17 & 9 & 6 & 2 & MGFA-PIS \\
\hline 10 & S. Jing & 2019 & Rituximab & 15 & 13 & 1 & 1 & QMG, MGFA-PIS, MG-ADL \\
\hline 11 & R. Topakian & 2019 & Rituximab & 56 & 39 & 14 & 3 & MGFA-PIS \\
\hline 12 & O. Landon-Cardinal & 2018 & Rituximab & 11 & 11 & 0 & 0 & QMG \\
\hline 16 & K. Rejdak & 2020 & Cladribine & 13 & 13 & 0 & 0 & MGC \\
\hline
\end{tabular}

*Study 5 was the open-label extension of Study 4. AChR, acetylcholinergic receptor; Musk, muscle-specific tyrosine kinase; MG-ADL, Myasthenia Gravis-Activities of Daily Living; MGC, Myasthenia Gravis Composite; MGFA-PIS, Myasthenia Gravis Foundation of America post-intervention status; MMT, manual muscle test; QMG, Quantitative myasthenia gravis.

standard mean difference of eculizumab was 1.409 (95\% CI: 0.556-2.262). The estimated standard mean difference of tacrolimus and cladribine were 0.923 (95\% CI: $0.297-1.548$ ) and 1.66 (95\% CI: $0.64-2.68)$, respectively. However, a small-study effect was found by Egger's test $(p<0.001)$.

Six of the included studies had reported MG-ADL results (Supplementary Figure 1). The study by Jing et al. failed to provide the SD of the change of MG-ADL score. It was imputed with the correlation coefficient as 0.5 . Fixed effect model was used for quantitative synthesis. The effect size was calculated with standard error. The pooled mean difference of rituximab was 4.400 (95\% CI: 2.610-6.190). The pooled mean difference of tacrolimus was 3.330 (95\% CI: 1.839-4.821). The pooled mean difference of eculizumab was 4.344 (95\% CI: 3.944-4.744). No small-study effect was found by Egger's test $(p=0.706)$.

Five of the included studies had provided the percentage of patients with MGFA-PIS as minimal manifestations (MM) (Figure 3). A random-effect model was used for quantitative synthesis. The estimated MM rate of rituximab was $67 \%$ (95\% CI: 0.40-0.89), while the estimated MM rate of eculizumab was $49 \%$ (95\% CI: $0.40-0.58)$. No small-study effect was found by Egger's test $(p=0.570)$. In addition, after the removal of Musk antibody-positive patients, the estimated MM rate of rituximab became $61 \%$ (95\% CI: 0.28-0.90).

\section{Efficacy in Preventing Worsening or Fluctuation}

The incidence density of MG exacerbation and crisis was estimated with a random effect model. The estimated incidence density of MG exacerbation for rituximab was 0.178 per patientyear (95\% CI: 0.099-0.319). The estimated incidence density of MG exacerbation for eculizumab was 0.218 per patient-year $(95 \%$ CI: 0.182-0.262) (Figure 4A). No small-study effect was found
TABLE 2 | Clinical characteristics of patients treated with rituximab, eculizumab, tacrolimus, and cladribine.

\begin{tabular}{lcccc}
\hline Therapy & Rituximab & Eculizumab & Tacrolimus & Cladribine \\
\hline No. of studies & 8 & 5 & 1 & 1 \\
Patients & 196 & 170 & 24 & 13 \\
Gender, female, $\%$ & $63 \%$ & $65 \%$ & $67 \%$ & $38 \%$ \\
Age at treatment & $49.3 \pm 15.97$ & $48.6 \pm 16.59$ & $43.8 \pm 13.20$ & $60.8 \pm 18.59$ \\
AChR-ab+ & 138 & 170 & 18 & 13 \\
Musk-ab+ & 43 & 0 & 0 & 0 \\
Seronegaitve & 15 & 0 & 6 & 0 \\
\hline
\end{tabular}

$A b$, antibody; AChR, acetylcholine receptor; Musk, muscle-specific tyrosine kinase.

by Egger's test $(p=0.822)$. The estimated incidence density of crisis for rituximab was 0.059 per patient-year (95\% CI: 0.0490.072 ). The estimated incidence density of crisis for eculizumab was 0.026 per patient-year (95\% CI: 0.011-0.062) (Figure 4 B). No small-study effect was found by Egger's test $(p=0.390)$.

\section{Common Adverse Events of Immunotherapies}

All included studies except for Brauner et al. reported adverse therapy events on patients with refractory MG. The incident density of adverse events and serious adverse events were calculated. A random-effect model was used for quantitative synthesis. The estimated adverse event density of rituximab was 0.134 per patient-year ( $95 \%$ CI: $0.064-0.281$ ), while the estimated adverse event density of eculizumab was 1.195 per patient-year (95\% CI: 0.635-2.248) (Figure 5). Tacrolimus and cladribine estimated adverse event densities at 0.292 per patient-year $(95 \%$ 


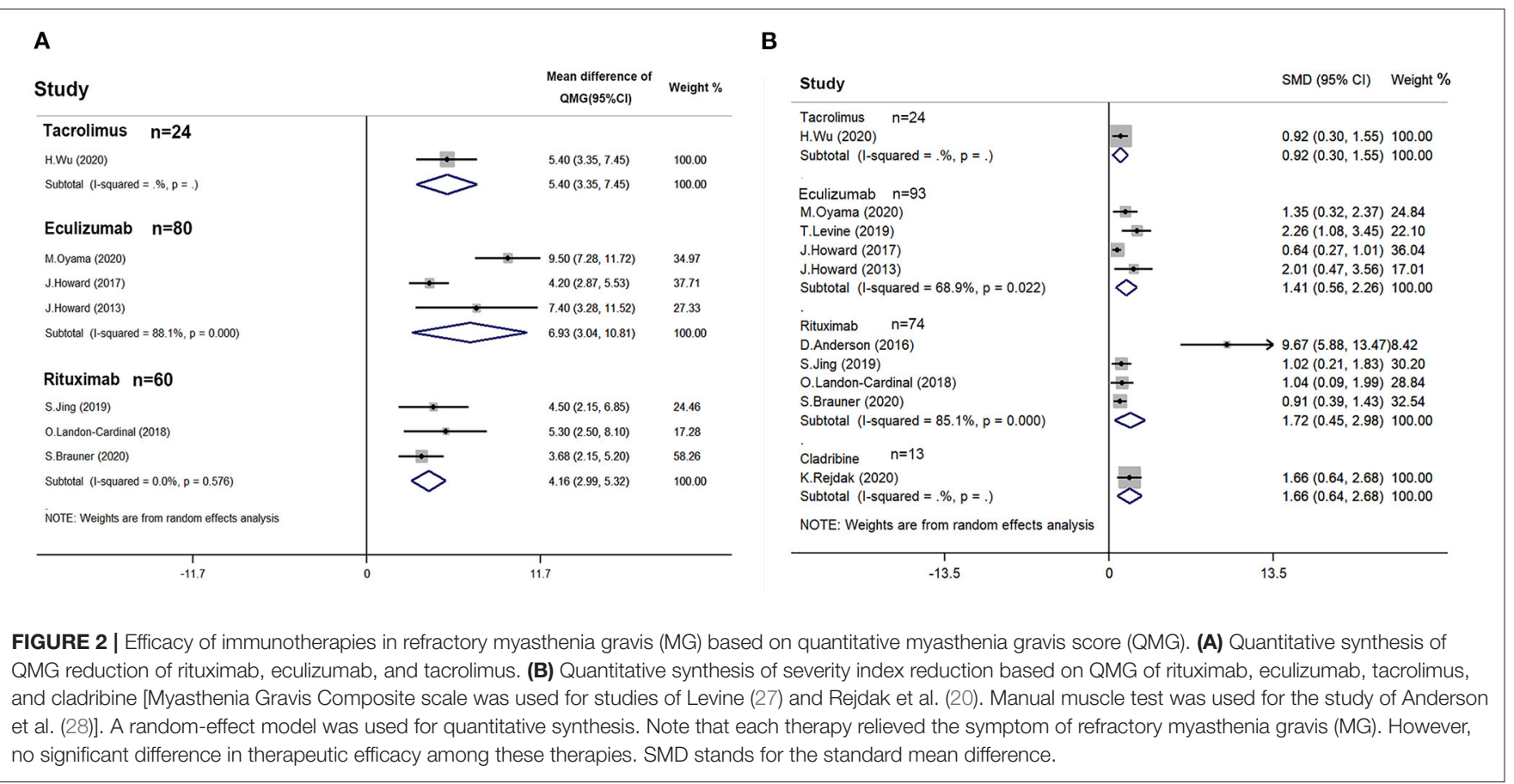

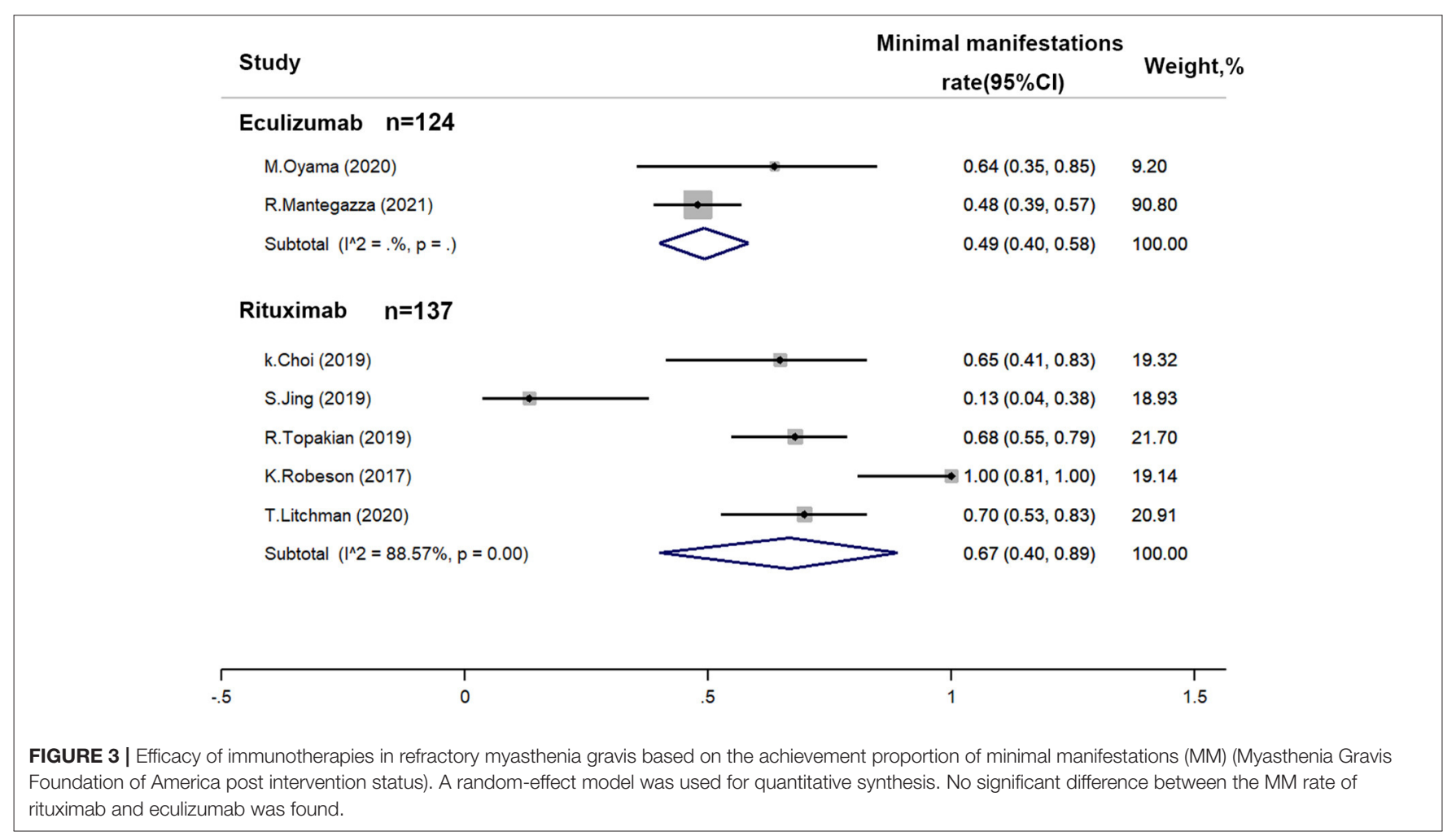

CI: $0.210-0.404)$ and 0.308 per patient-year (95\% CI: $0.115-$ $0.820)$, respectively. A small-study effect was found by Egger's test $(p=0.009)$.

Common adverse events of eculizumab include headache (18\%, 95\% CI: 0.04-0.36), nausea (14\%, 95\% CI: 0.09-0.20), myalgia (11\%, 95\% CI: 0.06-0.17), nasopharyngitis (8\%, 95\% CI: 0.00-0.27), upper respiratory tract infection (7\%, 95\% CI: $0.00-$ $0.21)$, and diarrhea (3\%, 95\% CI: 0.00-0.17). Common adverse events of rituximab include upper respiratory tract infection (1\%, 95\% CI: $0.00-0.06)$, infusion reaction (2\%, 95\% CI: $0.00-0.05)$, 


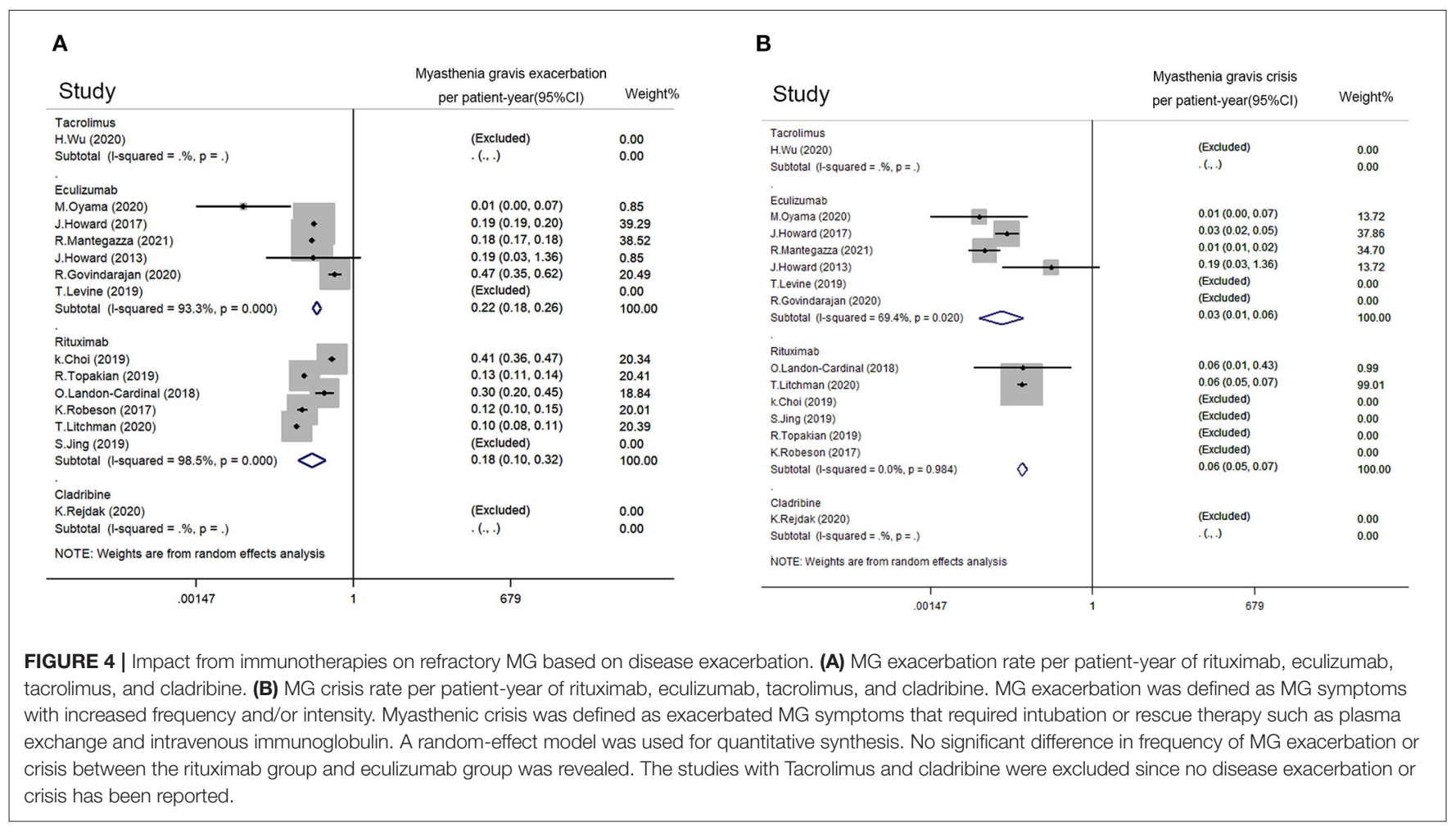

herpes zoster infection (1\%, 95\% CI: $0.00-0.04)$, and enteritis (1\%, 95\% CI: 0.00-0.04) (Supplementary Figure 2A).

The estimated serious adverse event density of rituximab was 0.082 per patient-year ( $95 \%$ CI: $0.035-0.190$ ), while the estimated adverse event rate of eculizumab was 0.281 per patient-year ( $95 \%$ CI: $0.146-0.540$ ) (Supplementary Figure 2B). No small-study effect was found by Egger's test $(p=0.058)$.

\section{Comparison of Low-Dose and High-Dose Rituximab}

The rituximab regimens included in the study were different. The low-dose rituximab was defined as a dose lower than 375 $\mathrm{mg} / \mathrm{m}^{2}$ twice a month. Studies of low-dose rituximab included Choi et al. (29), Jing et al. (24), and Brauner et al. (21). Other regimens were considered as high doses. Studies of high-dose rituximab included Landon-Cardinal et al. (25), Robeson et al. (23), and Litchman et al. (22). The estimated MM rate of low-dose rituximab was smaller than that of high-dose rituximab (39\%, $95 \%$ CI: $22-57$ vs. $84 \%, 95 \%$ CI: 71-93, Figure 6A). The pooled adverse events rate of low-dose rituximab was 9\% (95\% CI: 122 ), and that of high-dose rituximab was $12 \%$ (95\% CI: $0-32$ )

(Figure 6B).

\section{DISCUSSION}

A subset of MG is refractory to standard immunosuppressive therapy, whereby appropriate treatment is still uncertain. A total of 13 observational studies and three controlled studies included in this study identified rituximab, eculizumab, tacrolimus, and cladribine as possible optimal treatments.
Eculizumab is a-C5 monoclonal antibody, which inhibits C5 cleavage (30) and prevents damage to the neuromuscular junction from complement cascade in $\mathrm{MG}$ patients with AChR antibody (31). Similarly, rituximab is a monoclonal antibody targeting $\mathrm{CD} 20$ antigen. It modulates B-cell activation and inhibits $\mathrm{AChR}$ antibodydependent cytotoxicity in MG $(32,33)$. Tacrolimus and cladribine are non-steroidal immunosuppressants. Tacrolimus inhibits $\mathrm{T}$ cells and dampens antibody production (34). Cladribine has a selective effect on B and $\mathrm{T}$ lymphocytes, sustaining reduction of peripheral lymphocytes (35).

The efficacy of rituximab (4) and eculizumab (36) in MG has been proven in previous meta-studies. However, their efficacy in refractory MG has not been investigated yet. This study included 170 patients treated with eculizumab and revealed its efficacy for refractory MG. Quantitative synthesis showed that eculizumab relieved the symptoms of the patients refractory MG (estimated reduction of QMG: 6.93, estimated reduction of MG-ADL 4.34, estimated MM rate:49\%, estimated incidence density of MG exacerbation: 0.218 per patient-year). Similar efficacy of rituximab was revealed in a study including 196 patients (estimated reduction of QMG: 4.16, the estimated reduction of MG-ADL 4.40, estimated MM rate: $67 \%$, estimated incidence density of MG exacerbation: 0.178 per patient-year). However, no significant difference between their efficacy was found. Similarly, no significant differences between the incident density of $\mathrm{MG}$ exacerbation and also the incident density of MG crisis were found. 


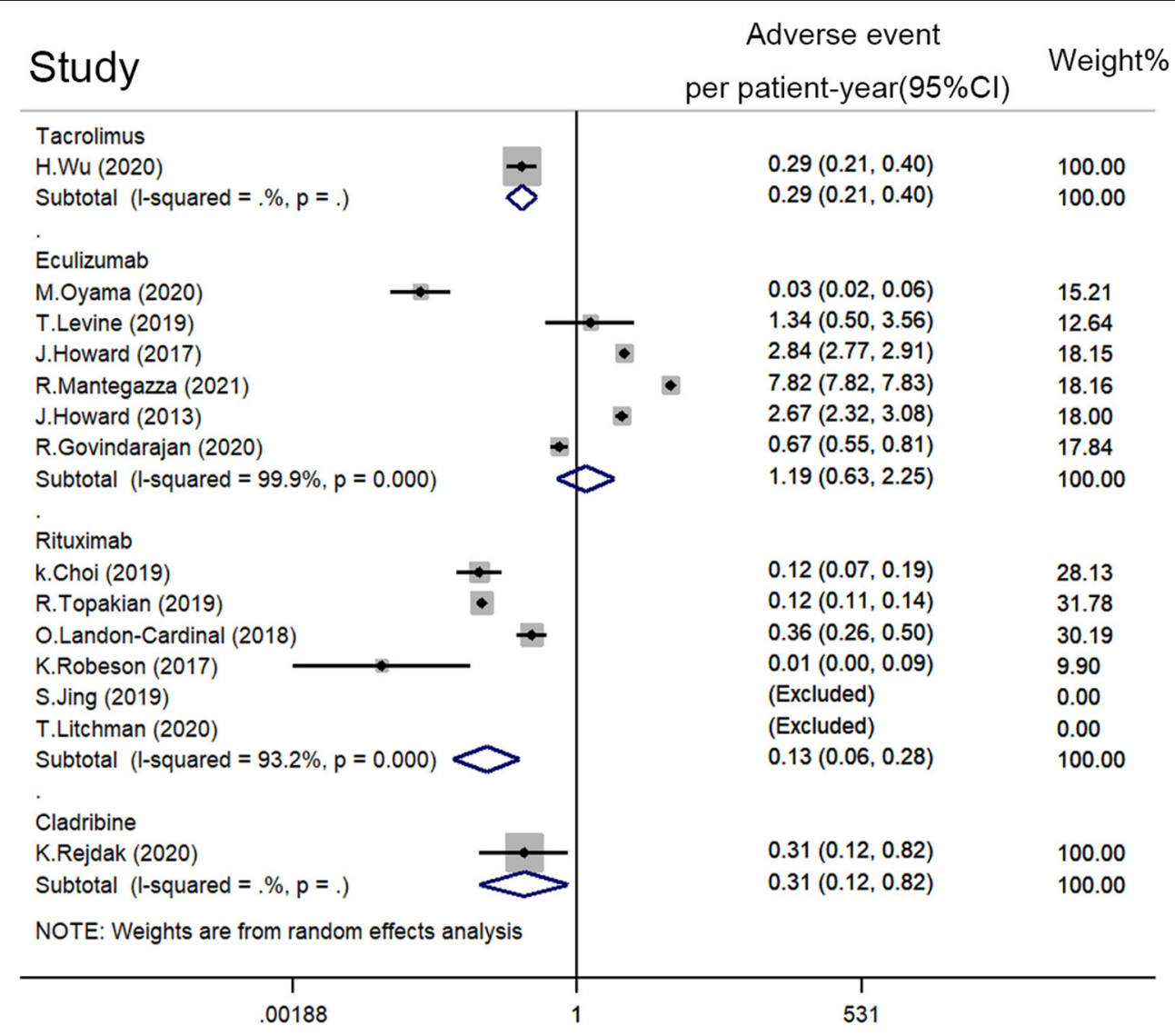

FIGURE 5 | Adverse event density (event rate per patient-year) of rituximab, eculizumab, tacrolimus, and cladribine on refractory MG. A random-effect model was used for the quantitative synthesis. Note that eculizumab had more adverse events than rituximab and tacrolimus. Besides, no significant difference in adverse event density between rituximab, tacrolimus, and cladribine was revealed.

A

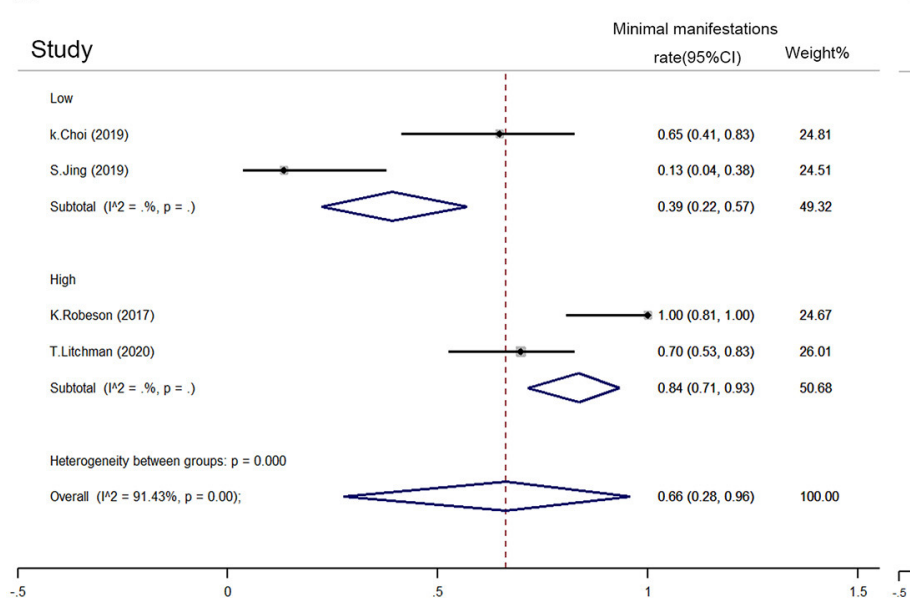

B

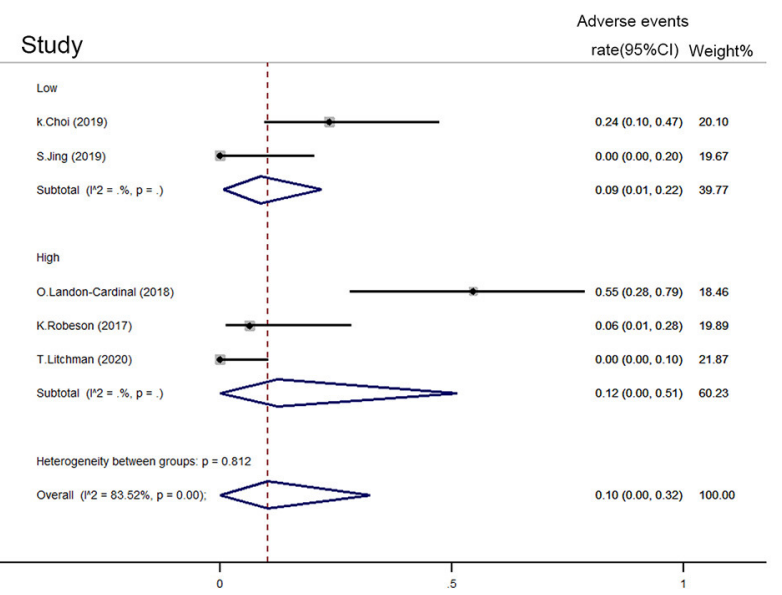

FIGURE 6 | Comparison between refractory MG subgroups with low-dose and high-dose rituximab. (A) Achievement proportion of MM (MG Foundation of America post intervention status) between low-dose and high-dose rituximab on refractory MG. (B) Adverse event density (event rate per patient-year) between low-dose and high-dose rituximab on refractory MG. High-dose rituximab was defined as a dose higher than $375 \mathrm{mg} / \mathrm{m}^{2}$ twice in a month and vice versa. A random-effect model was used for quantitative synthesis. The high-dose rituximab had better performance in achieving the MM rate than low-doses with similar adverse event density. 
The estimated adverse event density of eculizumab was more significant than that of rituximab (1.195 vs. 0.134 per patientyear). However, no significant difference in adverse severe event density was found between them. The most common adverse events of eculizumab were headache (18\%), nausea (14\%), and myalgia (11\%). The most common adverse events of rituximab were infusion reaction (2\%), upper respiratory tract infection (1\%), herpes zoster infection (1\%), and enteritis (1\%). Preventive non-steroid anti-inflammatory drugs may be considered for eculizumab treatment, and management of mild adverse events were important for adherence.

The efficacy and safety of low-dose and high-dose rituximab were also compared. High-dose rituximab was defined as a dose higher than $375 \mathrm{mg} / \mathrm{m}^{2}$ two times a month. High-dose rituximab had a higher MM rate than low-dose rituximab (84 vs. $39 \%$, Figure 6A), while no significant difference was observed between their adverse event rates (Figure 6B). In refractory MG, highdose rituximab appears to be a better regimen. However, the number of included rituximab studies was limited, and their regimens differed. A head-to-head comparison is needed to support the advantage of high-dose rituximab.

The efficacy of tacrolimus and cladribine was also revealed in this study; however, the number of included studies was insufficient.

These studies have several limitations. First, most of these were observational studies without control. The quality of those observational studies varied, and few high-quality studies were included. Second, the number of included patients was not enough, especially that of tacrolimus and cladribine. Moreover, the reported outcome of each study differed from each other, resulting in the insufficiency of the included patients in the quantitative synthesis. More controlled studies with standardized outcomes are necessary to search for the optimal treatment of refractory $\mathrm{MG}$.

There was a growing number of new options for treatment of refractory $\mathrm{MG}$, including neonatal Fc receptor blocking agents (37), Bortezomib [a proteasome inhibitor (38)], tocilizumab [blocker of interleukin-6 (39)], etc. However, few of them had been tested in trials of refractory MG. More well-designed clinical trials of these treatments on refractory MG and vigorous systemic reviews should be considered in order to establish an effective standardized treatment for patients with refractory MG.

This study revealed the efficacy and safety of eculizumab and rituximab in patients with refractory MG. Although few of the

\section{REFERENCES}

1. Bird SJ. Overview of the Treatment of Myasthenia Gravis. UpToDate (2020). Available online at: http://www.uptodate.com/contents/overview-ofthe-treatment-of-myasthenia-gravis

2. Schneider-Gold C, Hagenacker T, Melzer N, Ruck T. Understanding the burden of refractory myasthenia gravis. Ther Adv Neurol Disord. (2019) 12:1-16. doi: 10.1177/1756286419832242

3. Drachman DB, Adams RN, Hu R, Jones RJ, Brodsky RA. Rebooting the immune system with high-dose cyclophosphamide for treatment of refractory myasthenia gravis. Ann N Y Acad Sci. (2008) 1132:30514. doi: 10.1196/annals.1405.033 adverse events of eculizumab were serious, they were common to some degree during eculizumab treatment. Certain preventions may be necessary for better long-time adherence.

\section{DATA AVAILABILITY STATEMENT}

The raw data supporting the conclusions of this article will be made available by the authors, without undue reservation.

\section{AUTHOR CONTRIBUTIONS}

Article search was performed by XF. Full-text screening was performed by XF and YL. Quality evaluation was performed by $\mathrm{XF}$ and ZS. Data extract was performed by XF, MW, and WZ. Manuscript was written by XF, SL, and CZ. Study was designed by WZ. All authors contributed to the article and approved the submitted version.

\section{FUNDING}

This study was supported by grants from the National Key Research and Development Program (2018YFC1311304, 2018YFC1311300), the Southern China International Cooperation Base for the Early Intervention and Functional Rehabilitation of Neurological Diseases (2015B050501003), Guangdong Provincial Engineering Center for Major Neurological Disease Treatment, Guangdong Provincial Translational Medicine Innovation Platform for Diagnosis and Treatment of Major Neurological Disease, and Guangdong Provincial Clinical Research Center for Neurological Diseases. The sponsor or funding organization had no role in the design or conduct of this research.

\section{ACKNOWLEDGMENTS}

We would thank Alice Halim, MBBS, Zhongshan Hospital, Shanghai Medical College of Fudan University, and Carrie Law, MD, Shanghai Medical College of Fudan University.

\section{SUPPLEMENTARY MATERIAL}

The Supplementary Material for this article can be found online at: https://www.frontiersin.org/articles/10.3389/fneur. 2021.725700/full\#supplementary-material

4. Iorio R, Damato V, Alboini PE, Evoli A. Efficacy and safety of rituximab for myasthenia gravis: a systematic review and metaanalysis. J Neurol. (2015) 262:1115-9. doi: 10.1007/s00415-0147532-3

5. Howard JF, Utsugisawa K, Benatar M, Murai H, Barohn RJ, Illa I, et al. Safety and efficacy of eculizumab in anti-acetylcholine receptor antibodypositive refractory generalised myasthenia gravis (REGAIN): a phase 3, randomised, double-blind, placebo-controlled, multicentre study. Lancet Neurol. (2017) 16:976-86. doi: 10.1016/S1474-4422(17)30369-1

6. Mantegazza R, Antozzi C. When myasthenia gravis is deemed refractory: clinical signposts and treatment strategies. Ther Adv Neurol Disord. (2018) 11:1-11. doi: 10.1177/1756285617749134 
7. Wells G, Shea B, O'Connell D, Peterson J, Welch V, Losos M, et al. The Newcastle-Ottawa Scale (NOS) for Assessing the Quality of Nonrandomised Studies in Meta-Analyses. (2014). Available online at: http://www.ohri.ca/ programs/clinical_epidemiology/oxford.asp

8. Higgins J, Thomas J, Chandler J, Cumpston M, Li T, Page M, et al. Cochrane Handbook for Systematic Reviews of Interventions. 2nd ed. Chichester: John Wiley \& Sons.

9. Muppidi S, Utsugisawa K, Benatar M, Murai H, Barohn RJ, Illa I, et al. Long-term safety and efficacy of eculizumab in generalized myasthenia gravis. Muscle Nerve. (2019) 60:14-24. doi: 10.1002/mus.26447

10. Barohn RJ, McIntire D, Herbelin L, Wolfe GI, Nations S, Bryan WW. Reliability testing of the quantitative myasthenia gravis score. Ann N Y Acad Sci. (1998) 841:769-72. doi: 10.1111/j.1749-6632.1998.tb11015.x

11. Burns TM, Conaway M, Sanders DB. The MG composite: a valid and reliable outcome measure for myasthenia gravis. Neurology. (2010) 74:143440. doi: 10.1212/WNL.0b013e3181dc1ble

12. Sanders DB, Tucker-Lipscomb B, Massey JM. A simple manual muscle test for myasthenia gravis: validation and comparison with the QMG score. Ann NY Acad Sci. (2003) 998:440-4. doi: 10.1196/annals.1254.057

13. Wolfe GI, Herbelin L, Nations SP, Foster B, Bryan WW, Barohn RJ. Myasthenia gravis activities of daily living profile. Neurology. (1999) 52:14879. doi: 10.1212/WNL.52.7.1487

14. Jaretzki A, Barohn RJ, Ernstoff RM, Kaminski HJ, Keesey JC, Penn AS, et al. Myasthenia gravis: recommendations for clinical research standards. Neurology. (2000) 70:327-34. doi: 10.1212/wnl.55.1.16

15. Wu H, Wang Z, Xi J, Liu J, Yan C, Song J, et al. Therapeutic and immunoregulatory effects of tacrolimus in patients with refractory generalized myasthenia gravis. Eur Neurol. (2020) 83:500-7. doi: 10.1159/000510396

16. Oyama M, Okada K, Masuda M, Shimizu Y, Yokoyama K, Uzawa A, et al. Suitable indications of eculizumab for patients with refractory generalized myasthenia gravis. Ther Adv Neurol Disord. (2020) 13:19. doi: 10.1177/1756286420904207

17. Mantegazza R, Wolfe GI, Muppidi S, Wiendl H, Fujita KP, O’Brien FL, et al. Post-intervention status in patients with refractory myasthenia gravis treated with eculizumab during REGAIN and its open-label extension. Neurology. (2021) 96:e610-8. doi: 10.1212/WNL.0000000000011207

18. Howard JF, Barohn RJ, Cutter GR, Freimer M, Juel VC, Mozaffar T, et al. A randomized, double-blind, placebo-controlled phase II study of eculizumab in patients with refractory generalized myasthenia gravis. Muscle Nerve. (2013) 48:76-84. doi: 10.1002/mus.23839

19. Govindarajan R. Clinical experience with eculizumab in treatment-refractory acetylcholine receptor antibody-positive generalized myasthenia gravis. $J$ Neuromuscul Dis. (2020) 8:287-94. doi: 10.3233/JND-200584

20. Rejdak K, Szklener S, Korchut A, Baranowski D. Cladribine in myasthenia gravis: a pilot open-label study. Eur J Neurol. (2020) 27:586-9. doi: 10.1111/ene.14124

21. Brauner S, Eriksson-Dufva A, Hietala MA, Frisell T, Press R, Piehl F. Comparison between rituximab treatment for new-onset generalized myasthenia gravis and refractory generalized myasthenia gravis. JAMA Neurol. (2020) 77:974-81. doi: 10.1001/jamaneurol.2020.0851

22. Litchman T, Roy B, Kumar A, Sharma A, Njike V, Nowak RJ. Differential response to rituximab in anti-AChR and anti-MuSK positive myasthenia gravis patients: a single-center retrospective study. J Neurol Sci. (2020) 411:116690. doi: 10.1016/j.jns.2020.116690

23. Robeson KR, Kumar A, Keung B, DiCapua DB, Grodinsky E, Patwa HS, et al. Durability of the rituximab response in acetylcholine receptor autoantibody-positivemyasthenia gravis. JAMA Neurol. (2017) 74:60-6. doi: 10.1001/jamaneurol.2016.4190

24. Jing S, Lu J, Song J, Luo S, Zhou L, Quan C, et al. Effect of low-dose rituximab treatment on $\mathrm{T}$ - and B-cell lymphocyte imbalance in refractory myasthenia gravis. J Neuroimmunol. (2019) 332:216-23. doi: 10.1016/j.jneuroim.2019.05.004

25. Landon-Cardinal O, Friedman D, Guiguet $M$, Laforêt $P$, Heming N, Salort-Campana E, et al. Efficacy of rituximab in refractory generalized anti-AChR myasthenia gravis. J Neuromuscul Dis. (2018) 5:241-9. doi: 10.3233/JND-180300

26. Topakian R, Zimprich F, Iglseder S, Embacher $\mathrm{N}$, Guger $\mathrm{M}$, Stieglbauer $\mathrm{K}$, et al. High efficacy of rituximab for myasthenia gravis: a comprehensive nationwide study in Austria. $J$ Neurol.

(2019) 266:699-706. doi: 10.1007/s00415-019-09191-6

27. Levine TD. Safety of an abbreviated transition period when switching from intravenous immunoglobulin to eculizumab in patients with treatmentrefractory myasthenia gravis: a case series. Am J Case Rep. (2019) 20:96570. doi: 10.12659/AJCR.916424

28. Anderson D, Phan C, Johnston WS, Siddiqi ZA. Rituximab in refractory myasthenia gravis: a prospective, open-label study with long-term follow-up. Ann Clin Transl Neurol. (2016) 3:552-5. doi: 10.1002/acn3.314

29. Choi K, Hong Y-H, Ahn S-H, Baek S-H, Kim J-S, Shin J-Y, et al. Repeated low-dose rituximab treatment based on the assessment of circulating B cells in patients with refractory myasthenia gravis. Ther Adv Neurol Disord. (2019) 12:1756286419871187. doi: 10.1177/1756286419871187

30. Rother RP, Rollins SA, Mojcik CF, Brodsky RA, Bell L. Discovery and development of the complement inhibitor eculizumab for the treatment of paroxysmal nocturnal hemoglobinuria. Nat Biotechnol. (2007) 25:125664. doi: $10.1038 / \mathrm{nbt} 1344$

31. Tüzün E, Huda R, Christadoss P. Complement and cytokine based therapeutic strategies in myasthenia gravis. J Autoimmun. (2011) 37:13643. doi: 10.1016/j.jaut.2011.05.006

32. Townsend MJ, Monroe JG, Chan AC. B-cell targeted therapies in human autoimmune diseases: an updated perspective. Immunol Rev. (2010) 237:26483. doi: 10.1111/j.1600-065X.2010.00945.x

33. Boye J, Elter T, Engert A. An overview of the current clinical use of the anti-CD20 monoclonal antibody rituximab. Ann Oncol. (2003) 14:52035. doi: 10.1093/annonc/mdg175

34. Yoshikawa H, Kiuchi T, Saida T, Takamori M. Randomised, doubleblind, placebo-controlled study of tacrolimus in myasthenia gravis. J Neurol Neurosurg Psychiatry. (2011) 82:970-7. doi: 10.1136/jnnp-20 11-300148

35. Leist TP, Vermersch $\mathrm{P}$. The potential role for cladribine in the treatment of multiple sclerosis: clinical experience and development of an oral tablet formulation. Curr Med Res Opin. (2007) 23:2667-76. doi: 10.1185/030079907X233142

36. Wang L, Huan X, Xi JY, Wu H, Zhou L, Lu JH, et al. Immunosuppressive and monoclonal antibody treatment for myasthenia gravis: a network meta-analysis. CNS Neurosci Ther. (2019) 25:647-58. doi: 10.1111/cns. 13110

37. Wolfe GI, Ward ES, de Haard H, Ulrichts P, Mozaffar T, Pasnoor $\mathrm{M}$, et al. IgG regulation through FcRn blocking: a novel mechanism for the treatment of myasthenia gravis. J Neurol Sci. (2021) 430:118074. doi: 10.1016/j.jns.2021.118074

38. Khalesi N, Korani S, Korani M, Johnston TP, Sahebkar A. Bortezomib: a proteasome inhibitor for the treatment of autoimmune diseases. Inflammopharmacology. (2021) 29:1291306. doi: 10.1007/s10787-021-00863-2

39. Jonsson DI, Pirskanen R, Piehl F. Beneficial effect of tocilizumab in myasthenia gravis refractory to rituximab. Neuromuscul Disord. (2017) 27:565-8. doi: 10.1016/j.nmd.2017.03.007

Conflict of Interest: The authors declare that the research was conducted in the absence of any commercial or financial relationships that could be construed as a potential conflict of interest.

Publisher's Note: All claims expressed in this article are solely those of the authors and do not necessarily represent those of their affiliated organizations, or those of the publisher, the editors and the reviewers. Any product that may be evaluated in this article, or claim that may be made by its manufacturer, is not guaranteed or endorsed by the publisher.

Copyright (C) 2021 Feng, Song, Wu, Liu, Luo, Zhao and Zhang. This is an open-access article distributed under the terms of the Creative Commons Attribution License (CC $B Y)$. The use, distribution or reproduction in other forums is permitted, provided the original author(s) and the copyright owner(s) are credited and that the original publication in this journal is cited, in accordance with accepted academic practice. No use, distribution or reproduction is permitted which does not comply with these terms. 